\title{
Reproducing the Proximal Femur Anatomy Using Neck Anchorage Stem Design
}

\author{
Philippe Piriou and James Sullivan
}

\subsection{Introduction}

Neck-sparing stem designs enable a personalized (patient-specific) surgery by reproducing the native proximal femur anatomy. This facilitates physiological soft-tissue tension and hip kinematics, hopefully responsible for higher prosthetic hip function and patient satisfaction, as well as reduced risk of dislocation. Moreover, the bone economy achieved by this implant design is an obvious advantage in terms of easing revision surgery and decreasing stress-shielding-induced bone loss. The authors present their experience of using a neck-only tapered prosthesis with hydroxyapatite (HA) and porous coating: the Silent ${ }^{\mathrm{TM}}$ Hip system. The concept of the silent hip (Fig. 5.1) was first considered by Dr. Allan Ritchie in the mid-1990s when the need for a better solution for younger, more active and demanding patients was first recognised. Following this, a group of engineers and surgeons took this concept to development in conjunction with the University of Hamburg [1]. The implant went on to satisfy pre-clinical in vitro evaluation, and in 2003, the clinical study began to assess the stabil-

P. Piriou $(\bowtie)$

Clinique Ambroise Paré, Neuilly sur Seine, France

J. Sullivan

The Australian School of Advanced Medicine, Macquarie University Hospital,

Sydney, NSW, Australia ity of the implant, using two surgeons (Dr. Honl and Sullivan) to assist DePuy with the findings. Between January and November of 2003, 41 implantations were performed. Following this, a wider study began to test the validity of the technique with a wider range of surgeons, with encouraging results.

The reader might be surprised to read an article about an implant that is no longer marketed. The authors' experience with this implant was entirely satisfactory. It was unfortunately marketed in association with the large-diameter metal-on-metal bearings found to have high failure rates. The company, under the pressure of lawyers and regulators, decided to suddenly withdraw it when it had given excellent results. This innovation, for us, deserves to be reported until the concept is reborn in the future.

Healthy bone stock preservation at the time of primary total hip arthroplasty remains a goal for surgeons performing surgery on younger patients. The advent of short-stemmed femoral prostheses designed to conserve bone and load the femoral neck in a physiological way has enabled use in the general patient population requiring total hip arthroplasty. Indeed, the preservation of the elasticity of the proximal femur eliminates the proximal femoral stress shielding of conventional stems. Of principal benefit to the patient is that a subsequent revision of the prosthesis can potentially be made to a standard primary stem. Patients are often younger, more active and have 

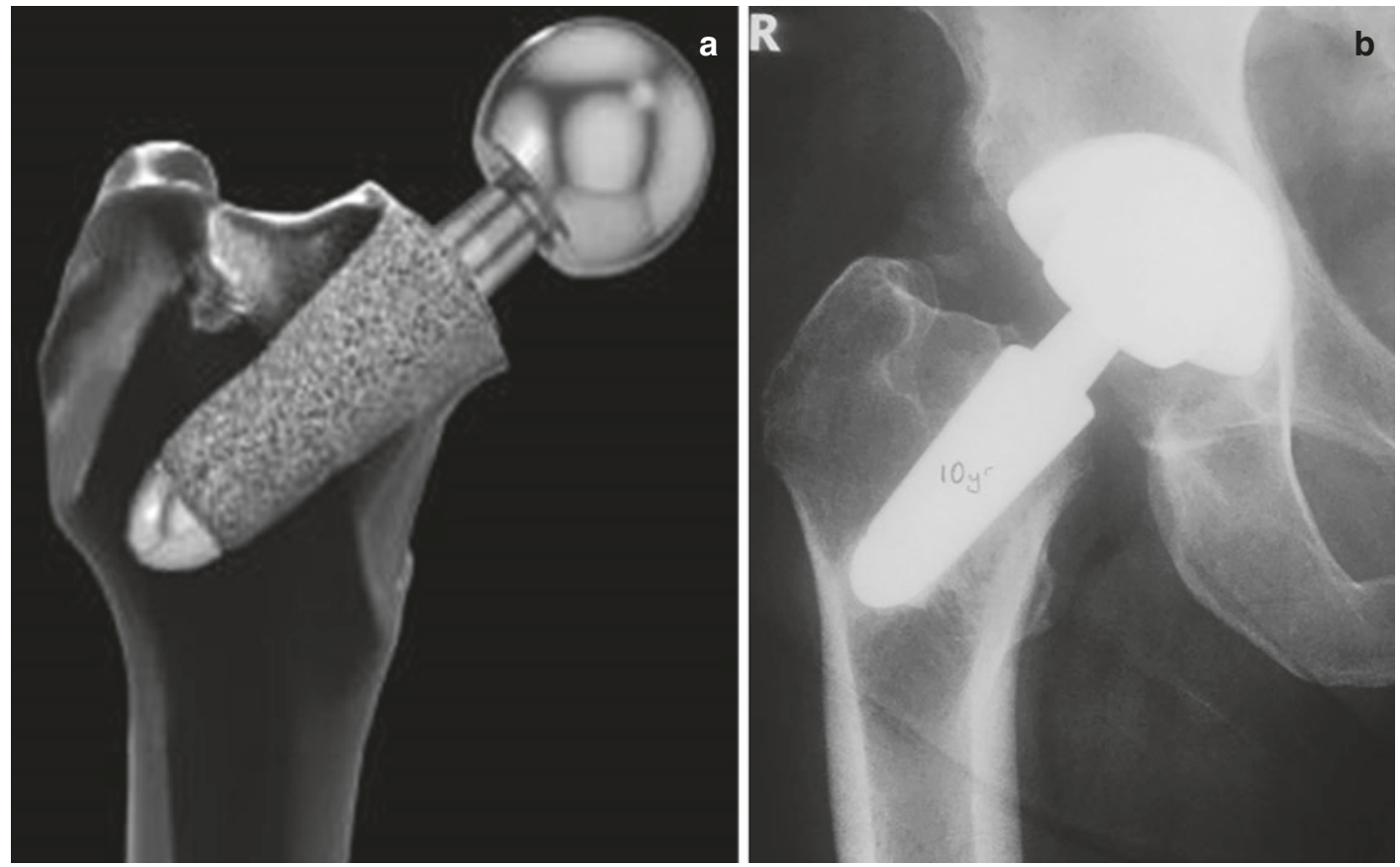

Fig. 5.1 The Silent ${ }^{\mathrm{TM}}$ stem is a neck-only tapered prosthesis with HA and porous coating (a). This stem is designed to load the calcar. (b) Illustrates a well-fixed silent ${ }^{\mathrm{TM}}$ stem at 10 years follow-up

increased expectations regarding function. As such, these patients are more likely to require a revision procedure.

\subsection{Design Rationale and Development of the Silent ${ }^{\text {TM }}$ Stem}

In the years 2000-2010, there existed on the market several types of femoral bone-conserving THA (Fig. 5.2) [2]: short-stemmed prostheses, neck-plate devices, neck-only stems and resurfacing. The thrust plate prosthesis (TPP) has been available since 1978. Now in its third generation of design, which has been in clinical use since 1992, it is made of titanium and has a coarse blasted surface to allow bony ongrowth. This third-generation TPP is reported to have improved survival and better functional outcomes than the second-generation TPP design. The Silent ${ }^{\mathrm{TM}}$ stem was born from the observation that sometimes, because of lateral thigh pain in the TPP, it was necessary to remove the side plate. In these cases, the implant in the centre of the neck continued to give good results.

Michael M Morlock and Matthias Honl in collaboration with Depuy's teams developed the Silent ${ }^{\mathrm{TM}}$ rod in its final version. First, there was a preclinical test phase to understand the biomechanics of the implant and to specify its conditions of use (Figs. 5.3 and 5.4). A press-fit implantation in good quality bone is essential to resist the varus forces and obtain sufficient stability to ensure that bony ongrowth is achieved. Regarding the surgical technique, the placement of the stem required an initial femoral head resection followed by neck cavity preparation with reaming. The final component is then implanted with a press fit.

\subsection{Clinical Data}

We report here the results of the first clinical study of the Silent ${ }^{\mathrm{TM}}$ implant. A cohort study was prospectively designed and carried out in two centres (M Honl-Germany, J Sullivan-Australia). The 

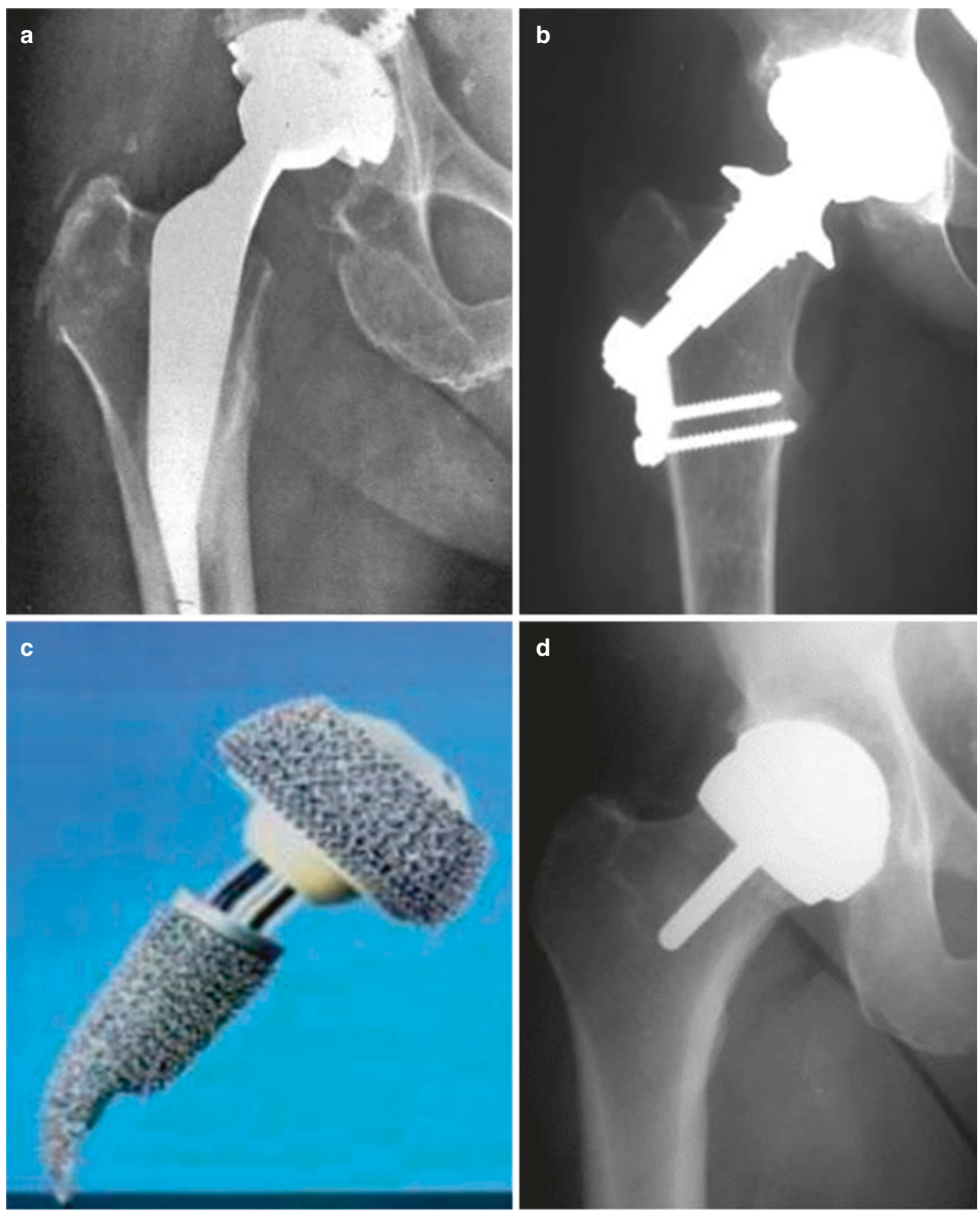

Fig. 5.2 Illustration of short stem (a), thrust plate (b), neck anchorage stem (c), and resurfacing (d) femoral component designs

outcomes of interest were a combination of clinical (occurrence of complications and functional assessment with Harris Hip and Oxford Hip scores) and radiographic (standard and RSA
X-rays at post-op, 3, 6, 12, 18, 24 and 60 months) measures. The Harris and Oxford Hip Scores were gathered pre-operatively and then at regular intervals over a 5-year period. The local research 


\section{- Stresses due to axial joint load component (Press Fit)}
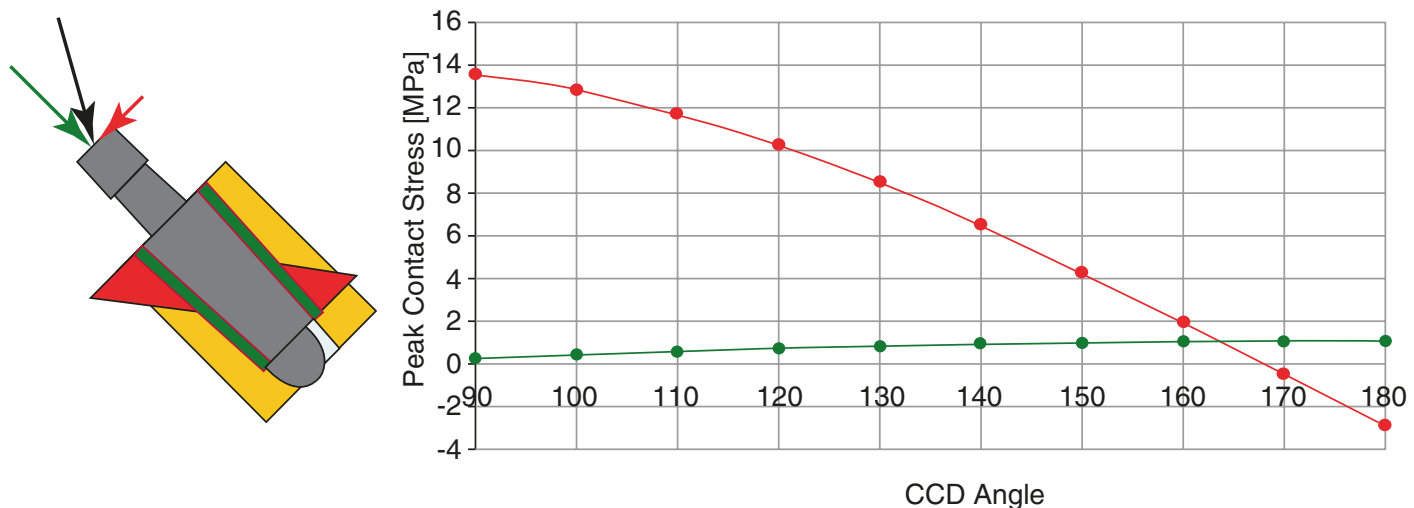

Axial load transfer is distributed $\rightarrow$ Low stresses

Toggling stresses are local $\rightarrow$ Higher stresses

Fig. 5.3 This figure shows the stress distribution on the femoral neck (left image) and the influence of the neckshaft angle (CCD angle) on the axial (green) and toggling (red) peak contact stress when the Silent ${ }^{\mathrm{TM}}$ stem is physiologically loaded (black arrow on left image)
Fig. 5.4 The

recommendations for implanting the Silent ${ }^{\mathrm{TM}}$ stem were to use the longest stem possible without lateral cortex contact in order to not reduce its press fit. This would maximise the stem-bone contact length and minimise stress on the calcar, therefore optimising stem osteointegration and reducing risk of peri-prosthetic fracture

- Long(est) stem and high(est) neck cut possible (without lateral cortex contact) $\rightarrow$ maximise stem-bone contact length $\rightarrow$ minimises bone interface stresses

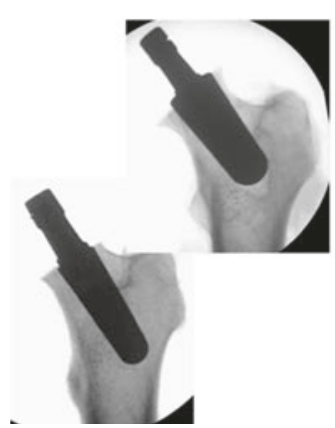

ethics committees and the regulatory authorities in both countries approved this study.

Patients with hip osteoarthritis (OA) between 25 and 65 years old and weighing less than $90 \mathrm{~kg}$ were included in this study. Significant bone loss or gross deformity of the femoral neck, osteonecrosis extending into the femoral neck, coxa vara (anatomical CCD angle of less than $125^{\circ}$ ), being $\mathrm{C}$ on the Charnley classification and subjects
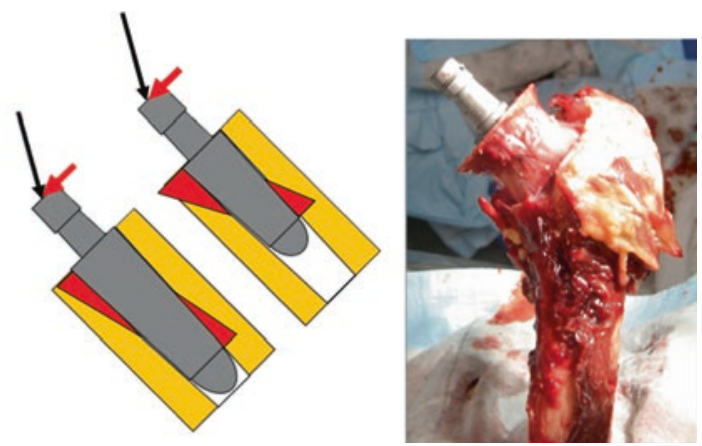

with inflammatory or Paget hip disease were criteria for exclusion.

Forty-one patients received a Silent ${ }^{\mathrm{TM}}$ Hip between January and November 2003, with additional tantalum beads inserted in the femur and attached to the stem for radio stereometric analysis (RSA). The characteristics of the cohort were as follows: mean age of 50.4 years (range 26-65), mean BMI of 26.6 (range 19-37) and 18 
females/23 males. The cause of the hip degeneration was primary OA in 28 cases, dysplasia in 3, avascular head necrosis in 6 , postinfection in 2 and 'other' in 2 cases. All patients received a ceramic-on-ceramic bearing except one with ceramic-on-polyethylene. The German group favoured the anterolateral approach with a $28-\mathrm{mm}$ femoral head, while the Australian group used a posterior approach with a $32-\mathrm{mm}$ head.

Five-year review was achieved with only one patient lost to follow-up. Good Harris and Oxford Hip Scores were obtained as illustrated on Figs. 5.5 and 5.6, respectively. Regarding the radiographic performance, no progressive femoral radiolucencies were observed; there was an increase of bone density in the calcar region
(Fig. 5.7). The RSA showed satisfactory stem migration over a period of 18 months (Fig. 5.8) demonstrating good primary stability and secondary fixation (osteointegration) of the Silent ${ }^{\mathrm{TM}}$ stem.

There were five reoperations involving the acetabulum but no revisions of the Silent stem; three cups were revised: one for recurrent dislocation, one following an early acetabular fracture and one for psoas impingement. One acetabular liner was exchanged as the ceramic liner fractured after the patient had a fall. Finally, one acetabular liner and femoral head were exchanged during washout procedure for an acute haematogenous periprosthetic infection occurring at 18 months post-op.
Fig. 5.5 Harris Hip Score pre-operatively and during 5-year follow-up
Fig. 5.6 Oxford Hip Score pre-operatively and during 5-year follow-up ( 0 best, 60 worst)
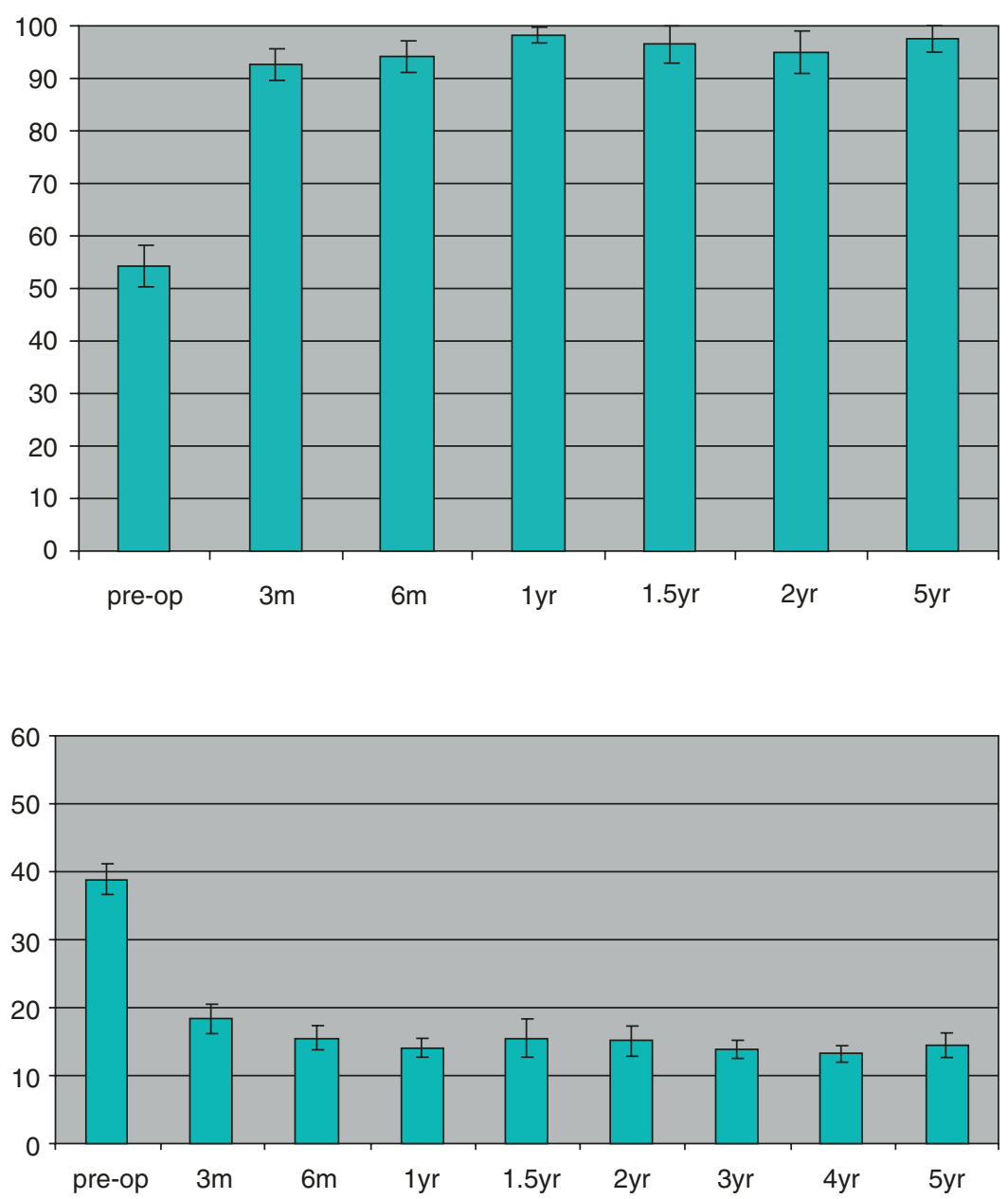

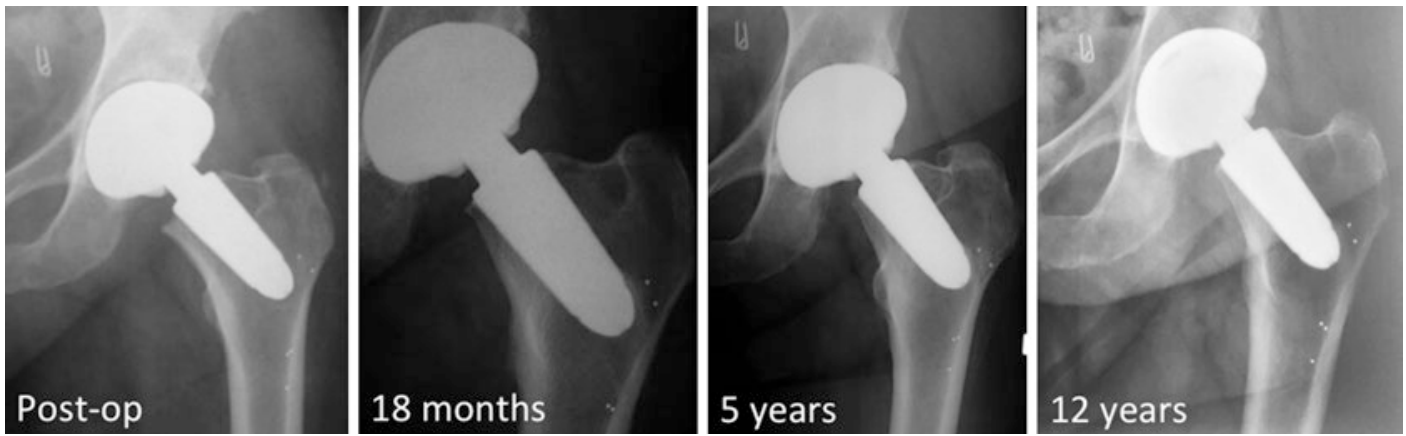

Fig. 5.7 Radiographic appearance of the bone remodelling around the Silent ${ }^{\mathrm{TM}}$ stem over a 12-year period

Fig. 5.8 This graphs shows the negligible migration of the Silent ${ }^{\mathrm{TM}}$ stems at 3,6, 12 and 18 months after implantation, as measured via RadioStereometric Analysis

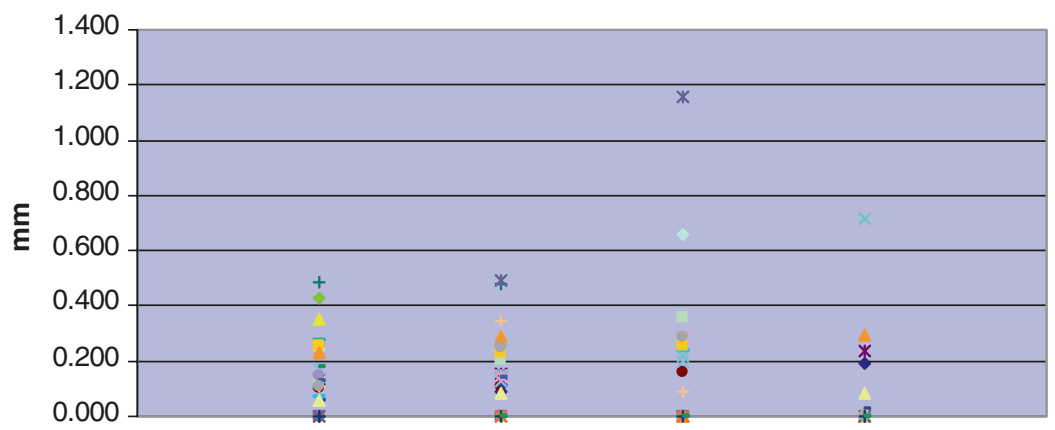

Follow-up time $3 \mathrm{~m} / 6 \mathrm{~m} / 12 \mathrm{~m} / 18 \mathrm{~m}$

\subsection{Discussion}

The silent stem is a bone-preserving implant that gets its anchorage in the femoral neck and so loads the proximal femur more physiologically than stemmed or short-curved implants. This is reflected by the bone remodelling [3] and preservation of the calcar as seen on radiographs [4]. Unlike the resurfacing procedure, the Silent ${ }^{\mathrm{TM}}$ stem can be used with destruction or deformity of the femoral head as it relies on fixation in the neck (Fig. 5.9). In addition, the resection of the femoral head enables easier access to the acetabulum for reaming and implantation of the acetabular component.

Previous RSA studies have suggested that distal intramedullary migration should be less than $1-1.5 \mathrm{~mm}$ in the first 2 years after implantation. The data for the Silent ${ }^{\mathrm{TM}}$ stem fell well within this limit, suggesting excellent stability of the stem. Preparation of the femur is performed by reaming. This creates an accurate bone defect for the tapered implant and would explain the excellent initial stability achieved.

The neck-sparing stem was a real innovation for young patients. It has proven its effectiveness in several clinical studies [5, 6]. It is unfortunate that the economic, political and regulatory climate has not given this stem an opportunity to demonstrate success on a wider platform. In any case, both authors regret it [7].

\section{Case Report}

The concept of using a short stem, anchored only in the femoral neck, was not exclusively developed by Depuy. This is evidenced by the following case of a 42-year-old man who was implanted by one of the authors with a Primoris stem. This stem was developed by Biomet and, similar to the Silent stem, fell into oblivion despite excellent preliminary results. The patient enjoyed running but had to stop due to right hip osteoarthritis 
Fig. 5.9 Leg Calve Perthes hip implanted with a Silent ${ }^{\mathrm{TM}}$ stem
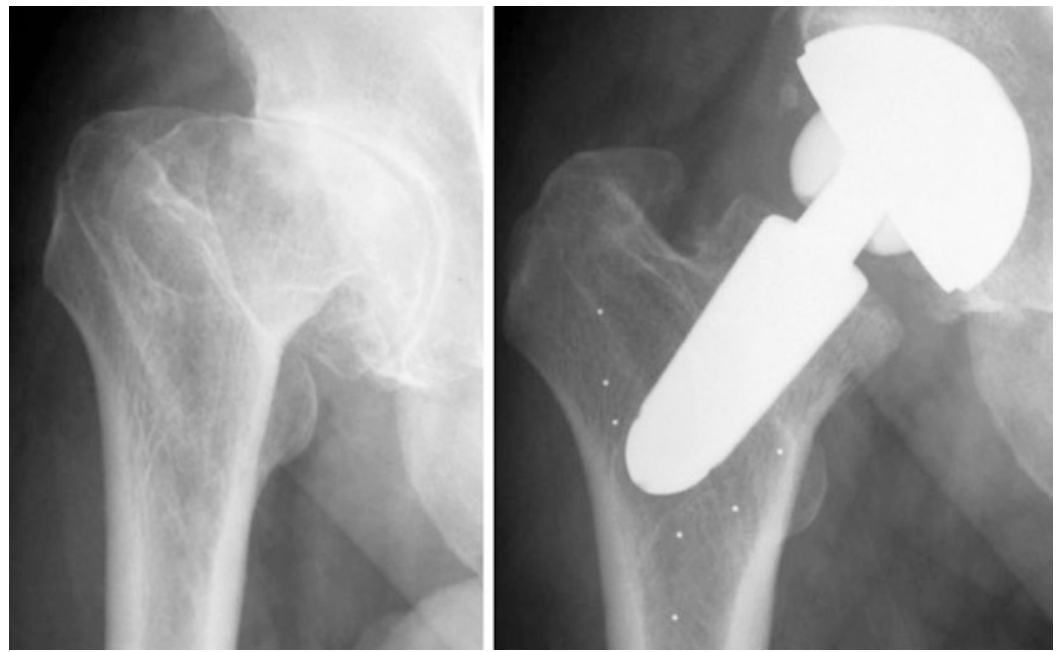

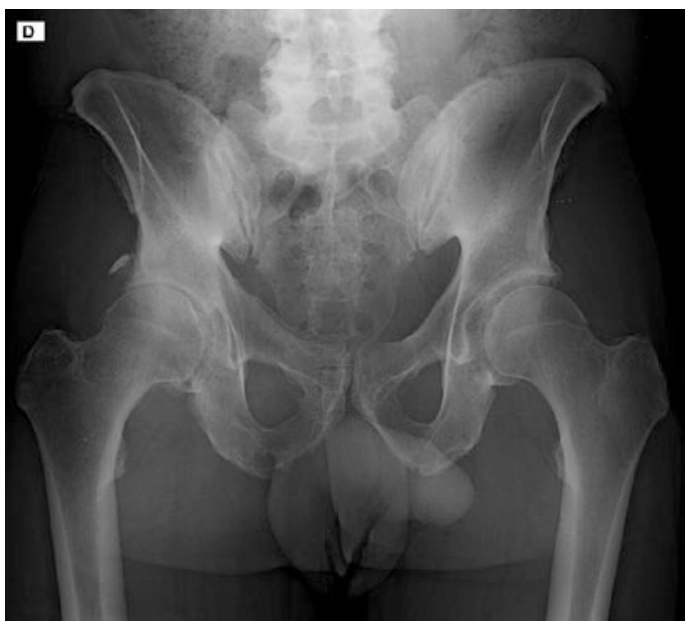

Fig. 5.10 Antero-posterior pelvic radiograph of a 42-year-old man showing right hip osteoarthritis

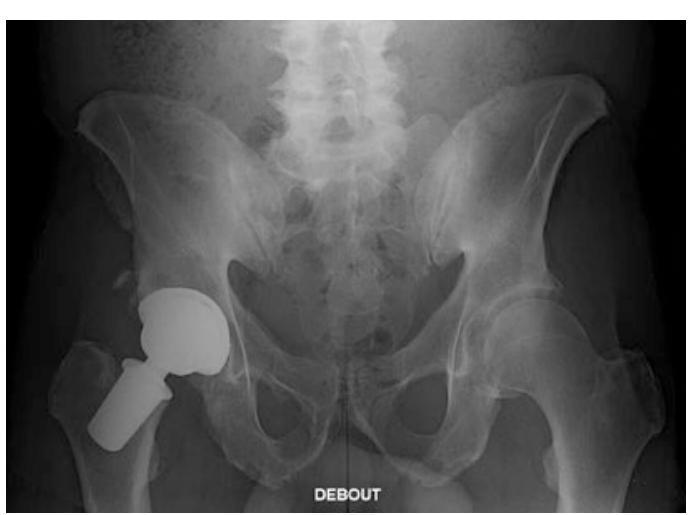

Fig. 5.11 Post-operative pelvic radiograph at 6 weeks

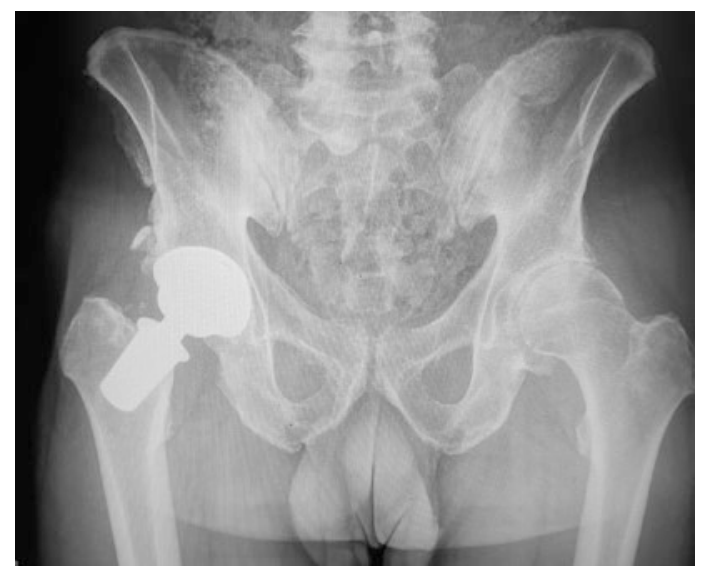

Fig. 5.12 Post-operative pelvic radiograph at 4 years, showing well-fixed components and no significant stress-shielding

(Fig. 5.10). After having undergone a total hip replacement through a direct anterior approach, the patient resumed his running activities as early as the sixth post-operative week (Fig. 5.11). At 4 years post-op, the patient continues to run with no detrimental effect on implant fixation (Fig. 5.12).

\section{References}

1. Falez F, Casella F, Papalia M. Current concepts, classification, and results in short stem hip arthroplasty. Orthopedics. 2015;38(3 Suppl):S6-13. 
2. Rajakulendran K, Field RE. Neck-preserving femoral stems. HSS J. 2012;8(3):295-303.

3. Tran P, Zhang BX, Lade JA, Pianta RM, Unni RP, Haw CS. Periprosthetic bone remodeling after novel shortstem neck-sparing total hip arthroplasty. J Arthroplast. 2016;31(11):2530-5.

4. Burchard R, Braas S, Soost C, Graw JA, Schmitt J. Bone preserving level of osteotomy in short-stem total hip arthroplasty does not influence stress shielding dimensions - a comparing finite elements analysis. BMC Musculoskelet Disord. 2017;18(1):343.
5. Hauer G, Vielgut I, Amerstorfer F, Maurer-Ertl W, Leithner A, Sadoghi P. Survival rate of short-stem hip prostheses: a comparative analysis of clinical studies and national arthroplasty registers. J Arthroplast. 2018;33(6):1800-5.

6. Molfetta L, Capozzi M, Caldo D. Medium term follow up of the biodynamic neck sparing prosthesis. Hip Int. 2011;21(1):76-80.

7. Giardina F, Castagnini F, Stea S, Bordini B, Montalti M, Toni A. Short stems versus conventional stems in cementless Total hip arthroplasty: a long-term registry study. J Arthroplast. 2018;33(6):1794-9.

Open Access This chapter is licensed under the terms of the Creative Commons Attribution 4.0 International License (http://creativecommons.org/licenses/by/4.0/), which permits use, sharing, adaptation, distribution and reproduction in any medium or format, as long as you give appropriate credit to the original author(s) and the source, provide a link to the Creative Commons license and indicate if changes were made.

The images or other third party material in this chapter are included in the chapter's Creative Commons license, unless indicated otherwise in a credit line to the material. If material is not included in the chapter's Creative Commons license and your intended use is not permitted by statutory regulation or exceeds the permitted use, you will need to obtain permission directly from the copyright holder. 